\title{
AsSESSING THE EFFECT OF UNICONDYLAR KNEE arthroplasty ON Proximal Tibia Bone STRAINS USING DigitAL IMAgE CORRELATION
}

\author{
Christopher J. Woods, Markus O. Heller and Martin Browne
}

Bioengineering Sciences Research Group, Faculty of Engineering and the Environment, University of Southampton, UK

\begin{abstract}
In order to develop computational models of implanted constructs to predict prosthesis performance, robust experimental tests need to be devised. In the case of unicondylar knee arthroplasty (UKA), where uptake of the procedure has been relatively low compared to traditional total knee arthroplasty techniques, computational modelling can give an insight into the factors affect the performance of UKA if verified with appropriate, preferably data rich, experimental simulations. In the present work, an image based strain analysis technique was applied for the assessment of the effect of UKA implantation on the strains developed in cortical bone of the proximal tibia. The results indicated the presence of increased strains in the proximal portion of the bone, which could be exacerbated in the case of poor implant positioning, or for patients with diminished bone quality.
\end{abstract}

\section{KEYWORDS}

Unicondylar Knee Arthroplasty, Orthopaedics, Implantation, Cadaver Bone, Strain, Digital Image Correlation

\section{INTRODUCTION}

Unicondylar knee arthroplasty (UKA) is thought to be a more effective solution than total knee arthroplasty (TKA) because of its improved functional outcome and the benefits of conservative tissue preservation coupled with minimally invasive surgical techniques [1]. It is considered suitable for more active patients with single-compartment knee disease, the mechanics of the knee are better preserved, and more functional anatomy is maintained [2]. UKA also has the advantage of rapid rehabilitation, short hospital stay and quicker recovery [3]. However, UKA is still underxploited as an alternative to TKA. This is related to a number of issues. For example, the National Joint Registry for England and Wales (NJR) 8th annual report showed that the risk of revision following UKA is higher than that for TKA. At 1 year post-op, revision rates of $0.58 \%$ for cemented TKA, $0.85 \%$ for uncemented TKA and $1.76 \%$ for UKA were reported. In addition, most surgeons are less familiar with UKA surgical technique than with TKA. In a survey to all 341 local members of the British Association for Surgery of the Knee, 95.5\% felt that a lack of familiarity or skill swayed them toward using a TKA and that patient selection needs to be improved [4]. The most prominent causes of UKA failure are polyethylene wear and implant loosening [5]. Aseptic loosening is believed to be due to excessive stress on the supporting cancellous bone [6]. The interface between the tibial component and the bone is much smaller than in TKA, so the underlying bone stresses are considered to be more sensitive to component positioning [7]. In order to understand the effect of implantation technique on the stresses generated beneath the implant, robust, data rich experimental methodologies need to be developed. Artificial polymer based bones are commonly used to investigate the effect of DOI: 10.5121/ijbes.2017.4301 
implantation on bone, however, while their geometries are representative of real bone, their mechanical behaviour is less representative (particularly the cancellous region) due to different time dependent properties and fracture behaviour. To this end, in the present study, cadaveric bone specimens are employed along with the digital image correlation method to assess strain development in UKA.

\section{Materials ANd Methods}

The current study evaluated the strains in a human cadaveric tibia using DIC in the unimplanted (here with designated 'intact') condition and after implantation of a UKA as described in detail below.

\subsection{Digital Image Correlation (DIC)}

Compared to traditional strain gauges, DIC allows full-field, non-contact, strain measurements. Using two cameras at a known angle to one another, the surface can be imaged in 3D and strain calculated from surface displacement vectors. In order to monitor displacement, the surface should have a random pattern that can be tracked easily. As the sample is increasingly loaded, images of the surface are acquired. Software tracks the relative displacement of the pattern during deformation between images by matching small areas or "subsets" containing pixels with unique grey values. A strain field is calculated by interpolating displacement data points at each subset centre, and these are smoothed to reduce noise. As DIC employs two cameras, if their relative angle is calibrated, a common 3D coordinate system can be determined, which allows limited outof-plane deformation to be measured.

\subsection{Cadaver Bone Preparation}

A right tibia (Anatomic gift register, Hanover, U.S.) of a 42-year-old Caucasian female was stripped of soft tissue (Figure 1a), the medial malleolus was removed, and the reference planes were identified. The distal $10 \%$ of the bone was potted using a custom made rig in a polymethylmethacrylate based potting compound (Technovit, Kulzer International, Hanau, Germany) so that it could be loaded as if in heel strike at 15 degrees flexion and a tibio-femoral angle of 177 degrees (Figure 1b). A drainage hole was left at the base of the test rig to prevent fluid pooling. The bone was dried with absorbent paper, and degreased with $70 \%$ ethanol prior to painting. The same bone was tested in the unimplanted and implanted state. To enable DIC testing, the intact bone had a speckle pattern applied on the surface. A selected region of interest was sprayed with thin uniform layers of matt white paint until the surface was completely covered. The speckle pattern was achieved by dipping toothbrush bristles into a small amount of black acrylic paint, then with the toothbrush perpendicular to the bone surface, the bristles were pushed backwards and released quickly, resulting in a random pattern of black speckles on a matt white background (Figure 1c).

Once the intact bone had been tested, it was implanted with a DePuy Sigma partial nee replacement, medial size 3, $8 \mathrm{~mm}$ tray (DePuy-Synthes, Warsaw, Indiana) in the neutral (idealised) position an tested again. By using the same bone, the same speckle pattern was used, and intra-specimen variability was minimised. 


\subsection{Mechanical Testing}

In both the intact and implanted case, the bone was placed in a specially constructed condylar test rig and loaded to achieve a 55:45 medial:lateral load distribution. The load was incrementally increased from zero to $1000 \mathrm{~N}$ (equivalent to approximately 1.4 times body weight). The load was limited to this level to ensure that permanent damage did not develop during testing, which could have influenced the resulting strains.

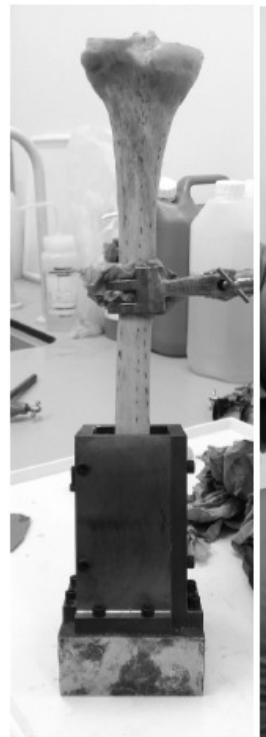

(a)

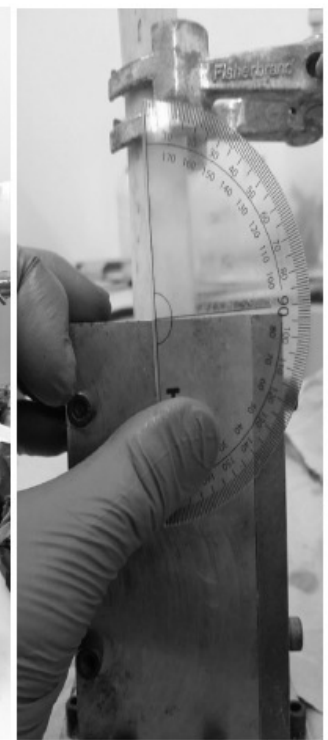

(b)

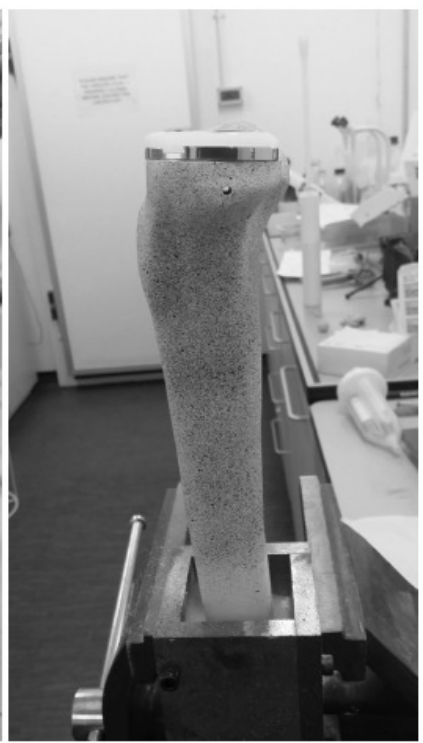

(c)

Figure 1. Preparation of cadaver tibia for digital image correlation - left to right - (a) tibia stripped of soft tissue, (b) aligned to simulate heel strike loading and (c) implanted with a speckle pattern applied to the surface prior to loading

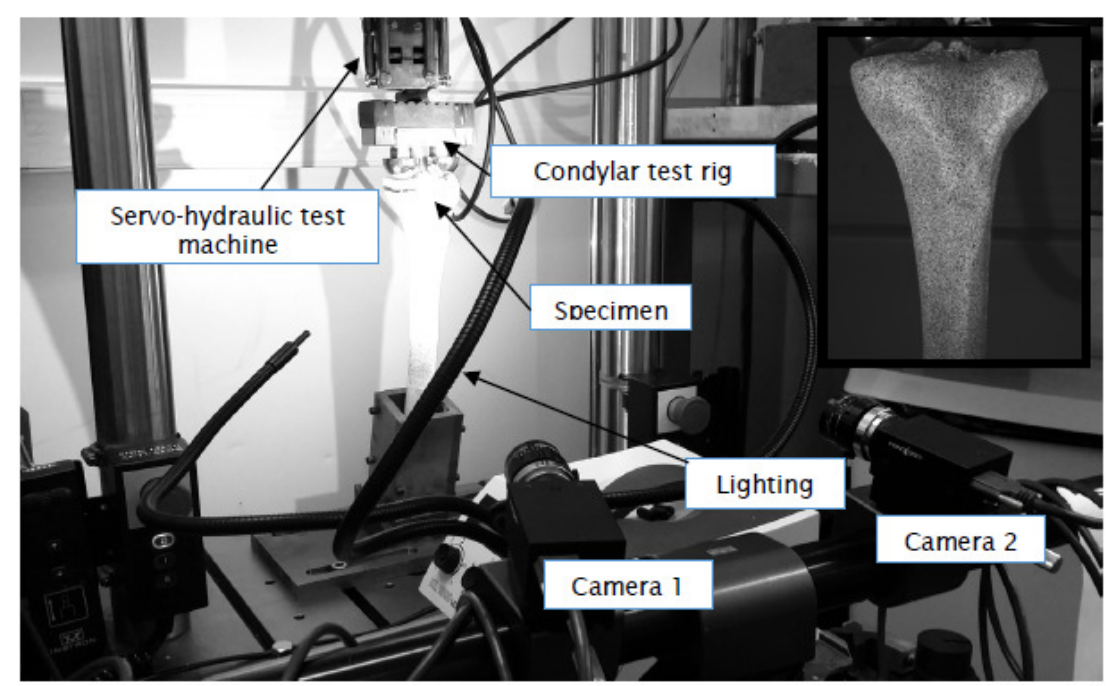

Figure 2. Digital image correlation test set up 


\subsection{Image Processing}

Images were acquired using two 5 megapixel cameras with a stereo angle of $20^{\circ}$ mounted on a rigid bar in line with the region of interest (Figure 2). It was ensured that the region of interest filled around $80 \%$ of the field of view. 40 calibration images were acquired for each test configuration and calibrated using stereo triangulation. An average difference between the theoretical and actual calibration images of 0.03 pixels was achieved. Five images were acquired at acquired at each load step at a frame rate of 10ms. The tests were repeated for two acquisition positions: anterior and medial.

Prior to testing under load, a set of unloaded, nominally zero-strain images were collected using the Vic-Snap (Correlated Solutions, South Carolina) capture system to quantify the system noise and error in rigid body movement correction. The specimen was then translated to assess the ability of the software to recognize that the speckle pattern has translated without deforming. This aims to account for any noise introduced by distortion from the camera lenses. A set of reference unloaded images were also taken after the test was complete to account for any settling effects of the bone in the potting medium. The load was held for at least 60 seconds at each load level to reduce any viscoelastic effects, which might impair the strain measurements.

For strain analysis, an unloaded speckle pattern was set as the reference image. The analysis area was defined and used for all load steps. The speckle pattern was sufficiently dense to allow using a small subset size ( $23 \times 23$ pixels); this ensured a high spatial resolution could be achieved. The correlation calculation providing access to the surface strain tensors was carried out using Vic-3D (Correlated Solutions, South Carolina) and raw strain maps were recorded.

\section{RESUlts AND DiscuSSION}

Mean noise levels of $~ 25$ microstrain were observed for DIC measurements on the unloaded intact bone.

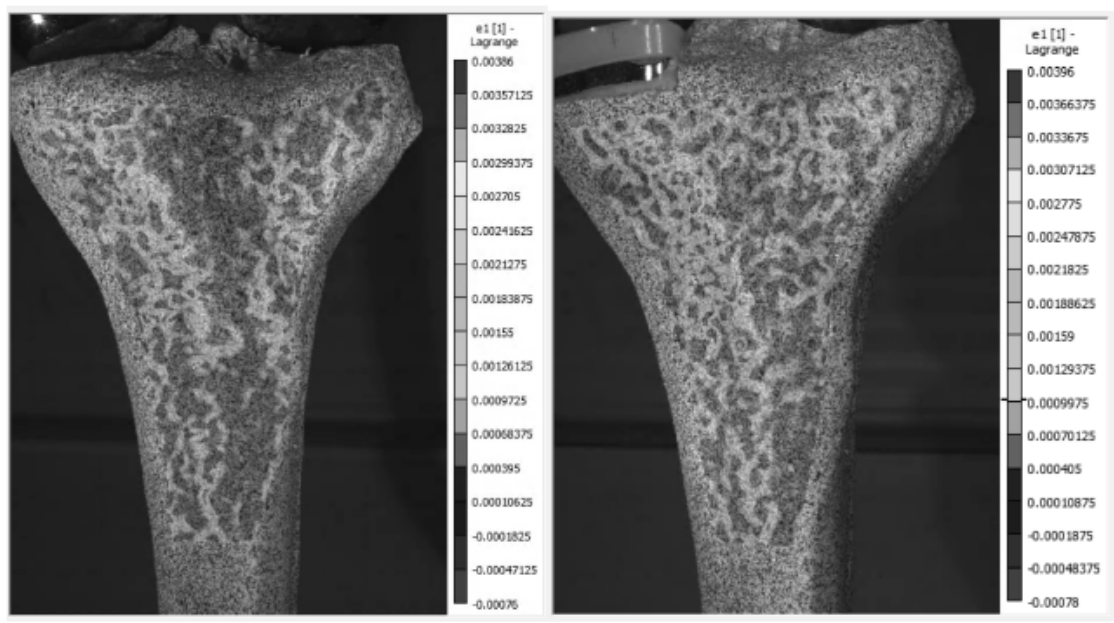

Figure 3. 1st principal strain of the intact (left) and implanted (right) knee under $1 \mathrm{kN}$ loading

Compared to the intact bone, the implanted bone experienced higher strains (represented by the lighter areas on the image) over a greater proportion of the bone surface, particularly in the proximal region (Figure 3). Mean strains in the intact and implanted bone at $1 \mathrm{kN}$ were $\sim 650$ and 
International Journal of Biomedical Engineering and Science (IJBES), Vol. 4, No. 3, July 2017

800 microstrain respectively, indicating that the presence of the implant increased the strains in the cortical, and by extension, the supporting cancellous bone. While these findings are in general agreement with previous work on artificial bone models [8], notably, there was less evidence of strain shielding in this cadaver model, potentially attributable to the use of a more representative test medium. Whilst synthetic bone models offer cheaper more reproducible alternative to native human bone and their use does not require ethical clearance, variations in cortical wall thickness, time dependent properties and internal structure exist between natural and synthetic bone, which could alter the structural response.

While UKA has the advantage of rapid rehabilitation, it is under-exploited as an alternative to TKA. The National Joint Registry for England and Wales (NJR) 8th annual report showed that the risk of revision following UKA is higher than that for TKA. However, long term success with very good clinical outcomes has also been noted [9]. This contrast in behaviour is also evident in the national knee register for Sweden, where the cumulative risk of revision for 10 UKA designs varied between 0.99 to 2.21 [10]. Increasingly, it is thought that UKA can be successful depending on the surgeon, the type of patient and the type of prostheses [10]. The present study appears to show that higher yet not deleterious bone strains are generated after implantation even under the most controlled conditions, where the cuts can be prepared accurately, there are no muscles or soft tissue to deal with, and the loading alignment is controlled. This effect of increased strain is likely to be exacerbated in the presence of implant misalignment, which could transpire with surgeons early on in the learning curve of UKA implantation. Further work will therefore investigate the effect of misalignment on bone strain.

\section{CONCLUSIONS}

The present work has demonstrated a framework for the experimental assessment of UKA performance in a cadaver based model. The experimental procedures described herein provide access to the detailed distribution of surface strains throughout the proximal tibia, which can be used to verify computational models, and assess the effect of component malpositioning. In this study, increased bone strains were observed in the presence of the implant. While the loads employed were relatively low compared to physiological loads $(\sim 3.5 \mathrm{x}$ body weight during walking), this relative increase in bone strain could be a contributor to unicondylar implant loosening as a result of everyday activities, particularly if the implant is misaligned.

\section{ACKNOWLEDGEMENTS}

The authors would like to express their thanks to Prof Luca Cristofolini and colleagues at the University of Bologna, Italy for sharing their expertise in cadaver testing, Mrs Sharon Coleman at the University of Southampton, UK for assistance in cadaver acquisition and preparation, and the Engineering and Physical Sciences Research Council (EPSRC) UK, Grant number EP/K034847/1, for financially supporting this work. DePuy International (Leeds, UK) are also acknowledged for supplying the UKA components used in this work.

\section{REFERENCES}

[1] Berger RA, Meneghini RM, Jacobs JJ, Sheinkop MB, Della Valle CJ, Rosenberg AG and Galante JO., (2005) Results of unicompartmental knee arthroplasty at a minimum of ten years of follow-up, J Bone Joint Surg Am, Vol. 87A No. 5, pp. 999-1006.

[2] Goodfellow J, O'Connor J, Dodd C and Murray D (2006) Unicompartmental arthroplasty with the Oxford knee, Oxford University Press. 194 pp.

[3] Newman JH, (2000) Unicompartmental knee replacement, Knee, Vol. 7 No. 2, pp. 63-70. 
[4] Schindler OS, Scott WN and Scuderi GR (2010) The practice of unicompartmental knee arthroplasty in the United Kingdom, J Orthop Surg (Hong Kong), Vol. 18, No. 3, pp. 312-319.

[5] Ashraf T, Newman JH, Desai VV, Beard D and Nevelos JE (2004) Polyethylene wear in a noncongruous unicompartmental knee replacement: a retrieval analysis, .Knee, Vol. 11, No. 3, pp.177-81.

[6] Taylor M and Tanner KE (1997) Fatigue failure of cancellous bone: a possible cause of implant migration and loosening, J Bone Joint Surg Br, Vol. 79, No. 2, pp. 181-182.

[7] Sawatari T, Tsumura H, Iesaka K, Furushiro Y and Torisu T (2005) Three-dimensional finite element analysis of unicompartmental knee arthroplasty-the influence of tibial component inclination, $\mathrm{J}$ Orthop Res., Vol. 23, No. 3, pp.549-554.

[8] Scott CEH, Eaton MJ, Nutton RW, Wade FA, Pankaj P and Evans SL (2013) Proximal tibial strain in medial unicompartmental knee replacements, Bone Joint J, Vol. 95-B, pp.1339-47.

[9] Tabor OB Jr, Tabor OB, Bernard M and Wan JY (2005) Unicompartmental knee arthroplasty: longterm success in middle-age and obese patients, J Surg Orthop Adv, Vol 14, No.2, pp. 59-63.

[10] Dunbar MJ (2007) Joint registries provide conflicting data on UKA outcomes, risk of revision, Orthopaedics Today Europe, Issue 1.

\section{AUTHORS}

Chris Woods obtained his undergraduate degree (BEng) in Mechanical Engineering from Oxford Brookes University, he went on to gain his Masters degree (MSc) in Bioengineering from the University of Southampton. After finishing his degree Chris worked collaboratively between Aurora Medical Ltd UK and the University of Southampton developing a novel dental implant concept. His experience includes computational bioengineering methods including statistical shape and intensity modelling and bone remodelling finite element analysis. He is currently looking at experimental verification of biomechanical models using digital image correlation and digital volume correlation. He is currently pursuing a $\mathrm{PhD}$ in Bioengineering Science.

Professor Markus O. Heller obtained a MSc in Aeronautical and Astronautical Engineering from the University of Stuttgart, Germany, a PhD in Human Biology from the University Ulm, and a Habilitation in Experimental Surgery from the Charite Universitätsmedizin Berlin, Germany. Since 2012 he is a Professor of Biomechanics at the University of Southampton, UK, in the Bioengineering Sciences Research Group within the Faculty of Engineering and the Environment. His research applies engineering principles to develop accurate and validated computational models of the mechanics of the human extremities, to understand subject specific loading conditions and assess the risk of mechanical overload of the human skeleton.

Professor Martin Browne obtained his Bachelor's degree in Materials Science from the University of Manchester, UK. After spending 3 years in industry researching carbon fibre composites for aerospace applications, he carried out his $\mathrm{PhD}$ in biomaterials science at the University of Southampton. He became a member of academic staff in 2003, and is now Head of the Bioengineering Sciences Research Group in the Faculty of Engineering at Southampton. His research interests include statistical analysis of implant performance, experimental characterisation of implants

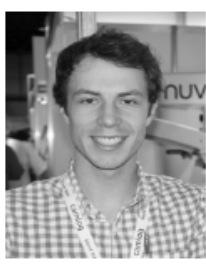
and biomaterials, and development of novel implant concepts in arthroplasty.
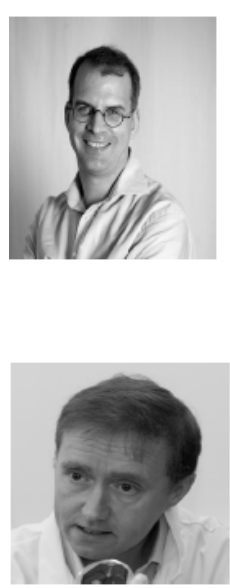